\title{
Microspheres of Mixed Proteins
}

\section{Ulyana Angel (Shimanovich), ${ }^{[a, b]}$ Devorah Matas, ${ }^{[b]}$ Shulamit Michaeli, ${ }^{[b]}$ Artur Cavaco-Paulo, ${ }^{[c]}$ and Aharon Gedanken*[a]}

\begin{abstract}
This paper describes the synthesis of mixed proteinaceous microspheres (MPMs) by the sonochemical method. The current fundamental research follows the research of Suslick and co-workers who have developed a method by which high-intensity ultrasound is used to make aqueous suspensions of proteinaceous microcapsules filled with water-insoluble liquids. ${ }^{[1]} \mathrm{By}$ using high-intensity ultrasound, we
\end{abstract}

have synthesized microspheres made of a few different proteins. The three proteins used in the current experiments are bovine serum albumin (BSA), green fluorescent protein (GFP), and cyan fluorescent protein-glucose bind-

Keywords: core-shell structures micelles $\cdot$ proteins $\cdot$ sonochemistry $\cdot$ surface modification

\begin{abstract}
ing protein-yellow fluorescent fused protein (CFP-GBP-YFP). The two synthesized microspheres made of mixed proteins are BSA-GFP and BSA-(CFPGBP-YFP). This paper presents the characterization of the sonochemically produced microspheres of mixed proteins. It also provides an estimate of the efficiency of the sonochemical process in converting the native proteins to microspheres.
\end{abstract}

\section{Introduction}

Ultrasonic emulsification is a well-known process and occurs in biphasic systems. Emulsification is necessary for microcapsule formation. Protein microspheres have a wide range of applications, including drug and oxygen delivery systems, ${ }^{[2-3,12]}$ contrast agents for sonography, ${ }^{[4]}$ and MRI. ${ }^{[5-7]}$

Micrometer-sized air-filled or liquid-filled proteinaceous microspheres (PMs) were synthesized from various kinds of proteins, such as bovine serum albumin (BSA) ${ }^{[8-10]}$ human serum albumin (HAS), ${ }^{[11]}$ and hemoglobin $(\mathrm{Hb})^{[12]}$ by the sonochemical method developed by Suslick and co-workers. In the late $1960 \mathrm{~s}$, a modified polymerization method for the

[a] U. Angel (Shimanovich), Prof. A. Gedanken

Department of Chemistry and Kanbar Laboratory

for Nanomaterials, Bar-Ilan University Center for

Advanced Materials and Nanotechnology

Bar-Ilan University, Ramat-Gan 52900 (Israel)

Fax: (+972)3-7384053

E-mail: gedanken@mail.biu.ac.il

[b] U. Angel (Shimanovich), Dr. D. Matas, Prof. S. Michaeli

Mina and Everard Goodman Faculty of Life Sciences

Bar-Ilan University, Ramat-Gan, 52900 (Israel)

Fax: (+972)3-7384053

[c] Prof. A. Cavaco-Paulo

Textile Engineering Department

University of Minho, 4800058 Guimaraes (Portugal)

Supporting information for this article is available on the WWW

under http://dx.doi.org/10.1002/chem.200902098. preparation of proteinaceous microspheres was developed by Rhodes, Scheffel, Wagner, and Zolle et al. ${ }^{[13-15]}$ The microsphere formation was accomplished by either heat denaturation at various temperatures or by cross-linking with carbonyl compounds in the ether phase. Other cross-linking agents, such as glutaraldehyde, were also used. Furthermore, air-filled human serum albumin microspheres were made by Dick and Feinstein ${ }^{[16,17]}$ as contrast agents in echosonography. However, these methods yielded microspheres with a short storage life, low microbubble stability, or high toxicity. The first liquid-filled proteinaceous microspheres were prepared by Suslick. They were made of BSA and were filled with n-dodecane, n-decane, n-hexane, cyclohexane, or toluene. The synthesis was conducted under a high-intensity ultrasonic probe, and $1.5 \times 10^{9}$ microcapsules per $\mathrm{mL}$ were obtained upon sonicating the precursor solution under air or $\mathrm{O}_{2}$. The average diameter of the $\mathrm{PM}$ was $2.5 \mu \mathrm{m}$ with a narrow size distribution (Gaussian distribution $= \pm 1.0 \mu \mathrm{m}$ ). The mechanism of the sonochemical formation of PM has been discussed previously. ${ }^{[18]}$ According to this mechanism, the microspheres are formed by chemically cross-linking cysteine residues of the protein with $\mathrm{HO}_{2}$ radicals formed around a micron-sized gas bubble or a nonaqueous droplet. The chemical cross-linking is responsible for the formation of the microspheres, and is a direct result of the chemical effects of ultrasound radiation on an aqueous medium.

In the current fundamental studies, we have extended Suslick's method and applied it to more than one protein, 
and have succeeded in forming mixed protein microspheres (MPMs). For this purpose we have used GFP (recombinant green fluorescent protein) ${ }^{[19]}$ CFP-GBP-YFP(cyan fluorescent protein, glucose binding protein, and yellow fluorescent fused protein), ${ }^{[20]}$ and BSA proteins as candidates for building mixed protein microspheres. It is worth mentioning that all the studied proteins have cys (cysteine) residues ${ }^{[21]}$ that are responsible for and capable of the formation of $\mathrm{S}-\mathrm{S}$ bonds (cross-linking) and facilitate the formation of the MPMs. Two of the three different proteins used in the current experiments, GFP and CFP-GBP-YFP, are fluorescent. $^{[22]}$ The GFP protein has a major excitation peak at a wavelength of $395 \mathrm{~nm}$ and a minor one at $475 \mathrm{~nm}$. Its emission peak is at $509 \mathrm{~nm}$, which is in the lower green portion of the visible spectrum. CFP-GBP-YFP was excited at $436 \mathrm{~nm}$, and the emission measured at $480 \mathrm{~nm}$. Three possible arrangements can be foreseen as originating from the assembly of two different kinds of proteins in forming microsphere structures. First, the combined two proteins together form the microsphere's walls. Second, the first protein forms the microsphere's walls, while the second protein is encapsulated inside the liquid-filled proteinaceous microsphere bubble. Third, each kind of protein forms separated oneprotein microspheres. To prove the arrangement of different proteins in a microsphere structure, we analyzed and characterized the products by light microscopy (Apo-Tome AxioImager.z1 microscope) ${ }^{[23]}$ and by DLS (dynamic light scattering) measurements. Microscopic observation was carried out for the microspheres to determine the arrangement of fluorescent proteins in MPM structures. In addition, the microscopic images (the z-stack images) were analyzed by the 'Imaris' software image analysis program, ${ }^{[24-25]}$ which provides a refined data set and, ultimately, a more precise evaluation of protein expression patterns. The 'Imaris' program enables one to flip and rotate the $\mathrm{z}$-stack in real time, and also to employ the Clipping Plane command that allows a microcapsule to be cut in half for internal review. This is very useful for investigating protein localization. The 'Imaris' program enables the possibility of localizing the fluorescent proteins GFP and CFP-GBP-YFP in the MPM structure.

MPMs have a potential application as a fluorescent detection of microspheres. In cell and molecular biology, the mixed protein microspheres could be introduced into organisms by endosythosis to follow the microspheres in the cell. In addition, since we have already demonstrated that drugs can be encapsulated in a PM in a 3 min sonication process, ${ }^{[26-27]}$ the fluorescence of the MPM can help in tracing the release of the drug upon the disintegration of the sphere. The modified forms of MPM (e.g., MPM, which consists of two different biologically active proteins) could be used as a biosensor. The mixed proteins can serve for the microscopic monitoring of proper targeting. We propose using a mixture of fluorescent protein with a nonfluorescing protein that contains a mutation. Since these proteins are found in the same microshpere if one is properly targeted the second goes along. In this way, we can assure that we targeted the protein to the proper nuclear locale. If we use a nuclear protein, we anticipate localizing the protein to the nucleus. The advantages of MPMs over the single PMs are as follows: 1) To make one PM of a fluorescent protein would be very expensive because of its price. On the other hand, to synthesize fluorescent microspheres (MPMs) we need a small amount of the expensive fluorescent proteins and a large amount of the less expensive nonfluorescent protein. 2) The MPM enables two parts to be targeted in the organism at the same time.

\section{Results and Discussion}

The formation, characterization, and properties of the sonochemically made PM were recently reviewed. ${ }^{[28]}$ The mechanism of the MPMs' formation is similar to the mechanism of PMs' formation. Aqueous sonochemistry caused by the implosive collapse of bubbles produces ${ }^{\circ} \mathrm{OH}$ and ${ }^{\circ} \mathrm{H}$. The radicals so produced form $\mathrm{H}_{2}, \mathrm{H}_{2} \mathrm{O}_{2}$, and, in the presence of $\mathrm{O}_{2}$, superoxide $\mathrm{HO}_{2}$. Hydroxyl, superoxide, and peroxide radicals are all potential protein cross-linking agents. The cysteine, which is present in BSA, CFP-GBP-YFP, and GFP proteins, is oxidized by the superoxide radical. The microcapsules are held together by protein cross-linking through disulfide linkages from cysteine oxidation.

Although we have previously shown that the microspherization of proteins can also happen in the absence of cysteines, ${ }^{[21]}$ in the case in which cysteine is not present, we had to lower the $\mathrm{pH}$ to form the spheres. In the current case, in which cysteines exist in the protein, under neutral $\mathrm{pHs}$, the $\mathrm{S}-\mathrm{S}$ bonds are the dominant factor stabilizing the sphere.

After the sonication, we could identify three different kinds of microspheres that were synthesized sonochemically: pristine BSA microspheres (one-protein microspheres), pristine (CFP-GBP-YFP)/GFP microspheres (one-protein microspheres), and BSA-(CFP-GBP-YFP)/BSA-GFP MPM (mixed protein microspheres). A schematic diagram of the arrangement and assembly of proteins leading to microsphere formation is presented in Figure 1.

The morphology of the microspheres was determined by using light microscopy (Apo-Tome AxioImager.z1 microscope). Figure 2 shows a micrograph depicting three kinds of microspheres: a) pristine BSA, b) BSA-(CFP-GBP-YFP), and c) BSA-GFP. As expected, the BSA microspheres are colorless and their average size was calculated to be $2.34 \mathrm{mi}-$ crons.

Figure $2 b$ shows the microspheres obtained upon the sonication of the BSA and CFP-GBP-YFP proteins. The figure does not represent the regular size of the blue microspheres. We have chosen to present microspheres that are much larger than the average blue microspheres obtained in the reaction, because these large microspheres exhibit a much stronger fluorescence signal than the BSA-(CFP-GBP-YFP) spheres. On the other hand, the color detected for the smaller spheres was much weaker. A strong blue sphere is observed at the center of each of the large two spheres. The 


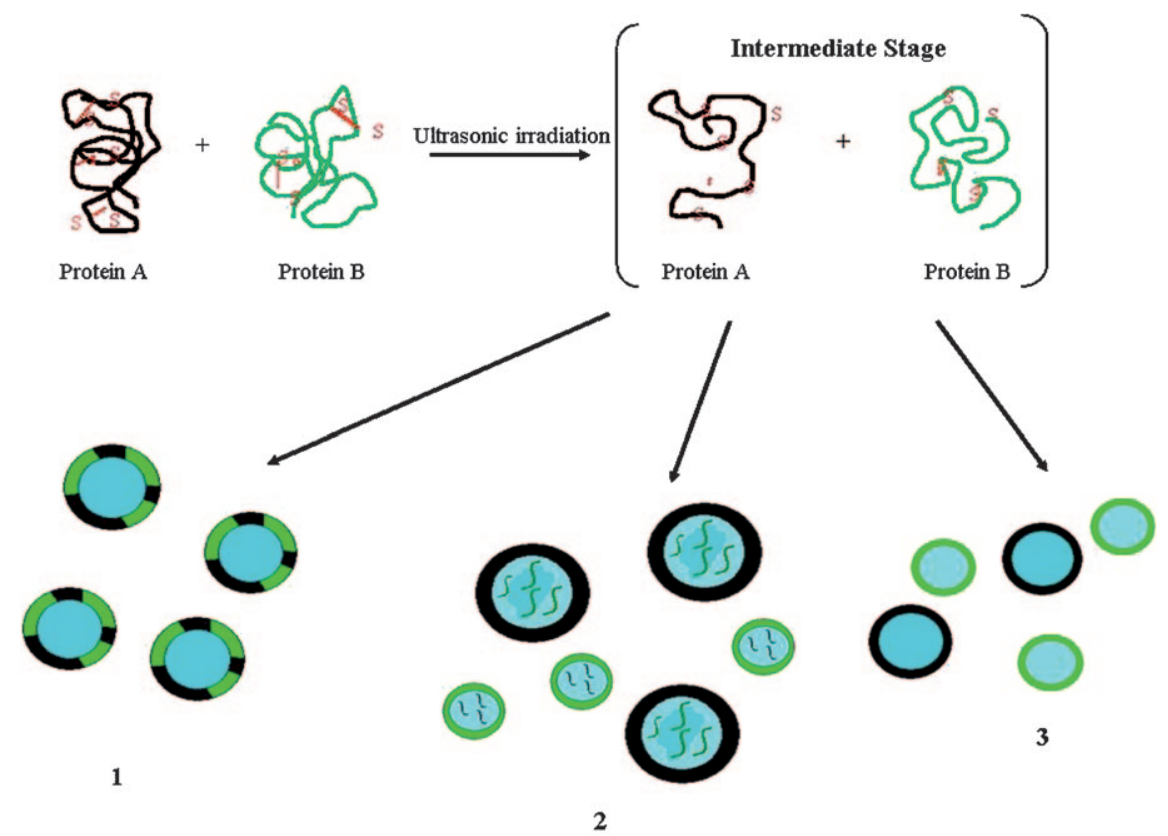

Figure 1. Schematic diagram of the arrangements and assembly of proteins leading to microsphere formation. $\mathrm{A}=$ any nonfluorescent protein; $\mathrm{B}=$ any fluorescent protein. 1) The combined two proteins together form the microsphere's walls. 2) The first protein forms the microsphere's walls, whereas the second protein is encapsulated inside the liquid-filled proteinaceous microsphere bubble. 3) Each kind of protein forms separated oneprotein microspheres.

larger of the two blue spheres is about 25 microns in size and the smaller one is 12 microns. These MPMs were microtomed for further studies. The small microspheres behind and above the blue spheres are black and are surrounded by a white corona. Since the microspheres have different sizes, it is not possible to focus the illuminating beam on all the particles simultaneously. Thus, the corona is a result of focusing the illuminating beam on the blue spheres, and when the beam is focused on the black spheres the corona is not detected. At this stage, without further analysis, it seems that the blue microspheres are attributed to the CFP-GBPYFP proteins or to the MPMs of BSA-(CFP-GBP-YFP), whereas the small black microspheres are due to BSA.

The microsphere size distribution of the three products (BSA, BSA-(CFP-GBP-YFP), and BSA-GFP) was examined by DLS measurements. When pristine BSA was sonicated, the DLS results yielded spheres with an average size of 2.34 microns (for BSA microspheres). When a mixture of BSA and GFP was sonicated, a bimodal size distribution was found in the DLS measurements. The average size for the major component of the BSA-GFP MPM was $1.406 \mu \mathrm{m}$ $(92.6 \%)$. The minor component of $7.4 \%$ showed an average size of $244 \mathrm{~nm}$.

Only one DLS peak is obtained for the products of the sonicated mixture of BSA-(CFP-GBP-YFP). The average size of the spheres was found to be $3.525 \mu \mathrm{m}$. The results of the calculated size distribution of the microspheres are presented in Figure 3. The picture indicates that tBSA-(CFPGBP-YFP) is the largest sphere, followed by the BSA spheres, and the BSA-GFP particles are the smallest. We at- tribute this order to the molecular weight of GFP and (CFPGBP-YFP) proteins. The molecular weight and the size of the GFP is the smallest, which makes the spheres smaller. This number is increased in the (CFP-GBP-YFP) protein, making the spheres larger than in BSA-GFP. We assign the minor component $(7.4 \%)$ in the BSA-GFP mixture with an average size of $244 \mathrm{~nm}$ to the GFP (one-protein) microspheres. In both mixtures, BSAGFP and BSA-(CFP-GBPYFP), a small amount ( 5\%) of pristine BSA-BSA (average size $\approx 2.3$ microns) microspheres are detected. Since their contribution to the size distribution is negligible, the size order depicted in Figure 3 is a true presentation of the microspheres' sizes.

The different ways of controlling the size of proteinaceous microspheres were previously studied..$^{[28]}$

To prove the arrangement of different proteins in the microsphere structure, we analyzed and characterized the products by light microscopy (Apo-Tome AxioImager.z1 microscope). We analyzed the Apo-Tome images by the 'Imaris' program to demonstrate the location of the fluorescent protein in the MPM structure. The 'Imaris' program enabled us to "cut" the microspheres into different planes ( $x y$, $x z$, and $y z$ planes), which allowed us to locate the different proteins in the microsphere structure (see the Supporting Information).

An example of a "reverse" BSA-(CFP-GBP-YFP) microsphere is shown in Figure 4a, in which the fluorescent CFPGBP-YFP protein was encapsulated inside the liquid-filled BSA microsphere bubble. Figure $4 \mathrm{~b}$ shows a BSA-(CFPGBP-YFP) mixed protein microsphere, in which the walls are a mixture of the two proteins, BSA and CFP-GBP-YFP. The light emitted from the microsphere's walls does not spread homogeneously over the whole surface and only patches of blue are observed. We can clearly identify fragments of the blue color originating from the MPM wall that are assigned to the fluorescent CFP-GBP-YFP protein. Figure $4 \mathrm{c}$ and $\mathrm{d}$ show three kinds of microspheres in the same solution, PM, MPM, and "reverse" MPM. Unlike Figure 4a, in which homogeneous fluorescence originated throughout the content of the sphere, Figure $4 \mathrm{~d}$ reveals many colored spheres in which the green color is located in only a small part of the volume. These kinds of spheres are clearly mixed spheres, in which the two proteins, BSA and GFP, construct the walls of the sphere. Figure 4c illustrates BSA-(CFP- 
a)

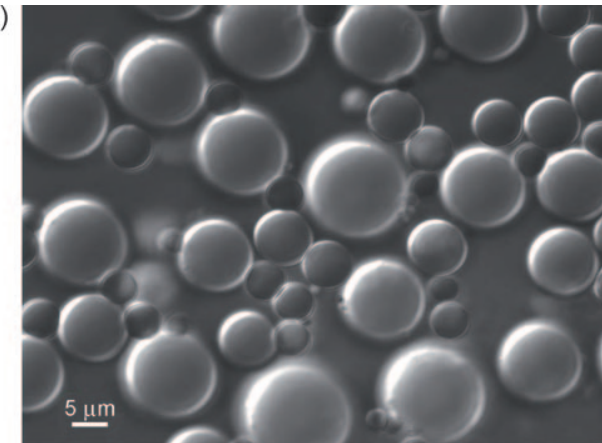

b)

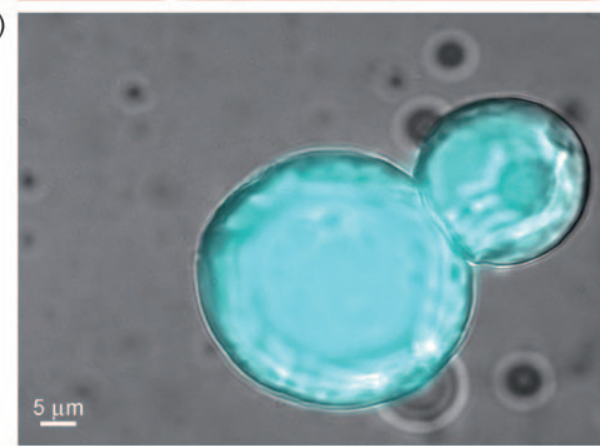

c)

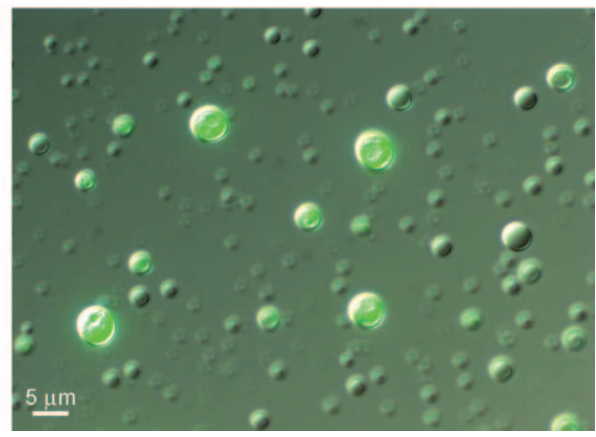

Figure 2. a) Apo-Tome image of BSA-BSA one-protein microspheres. b) Apo-Tome image of BSA-(CFP-GBP-YFP) MPM. c) Apo-Tome image of BSA-GFP MPM

GBP-YFP) MPMs and pristine BSA spheres. As stated previously, these BSA spheres are only $5 \%$ of the total spheres observed in our studies.
The calculated abundance of each kind of microsphere ("reverse" microspheres, BSA-(CFP-GBP-YFP)/BSA-GFP MPMs, pristine BSA, and pristine GFP/(CFP-GBP-YFP) spheres) was studied by using the 'Scion' image analysis program. These calculations have revealed differences in the particle size of the four kinds of the above-mentioned microspheres. To make this kind of analysis, we first need to identify each kind of microsphere. In "reverse" microspheres, in which the fluorescent (GFP/CFP-GBP-YFP) protein was encapsulated inside the liquid-filled BSA PM, the light (green for GFP or blue for CFP-GBP-YFP protein) was emitted from the inner part of the spheres. The BSA(CFP-GBP-YFP)/BSA-GFP MPMs emitted the light, which is not homogeneously spread, from the microspheres' walls. The pristine BSA spheres have no fluorescent signal. Pristine GFP or CFP-GBP-YFP PMs emitted the homogeneously spread green or blue light, respectively, from the PM walls.

Figures 5 and 6 present the percentages and average sizes of the different structures for MPMs of BSA-GFP and BSA-(CFP-GBP-YFP), respectively. Figure 5 is divided into $a$ and $b$, each emphasizing different microspheres. In Figure 5a, in which the green color dominates, it is easier to detect the microspheres with green patches or with a complete green wall. On the other hand, Figure $5 \mathrm{~b}$ emphasizes the more blackish colors and, therefore, helps to detect the pristine BSA microspheres. The results show that the major components of the BSA-GFP and BSA-(CFP-GBP-YFP) solutions after the sonication are mixed protein microspheres, namely, microspheres the skeleton of which is composed of the two proteins. Figure $5 \mathrm{c}$ shows the abundance of the different kinds of microspheres for the BSA-GFP solution. For this solution, four types of microspheres were created: a) pristine BSA microspheres $(3.6 \%)$ with an average size of $2.3 \mu \mathrm{m}, \mathrm{b})$ pristine GFP microspheres $(3.7 \%)$ with an average size of $435 \mathrm{~nm}$, c) reverse GFP-BSA MPMs $(8.4 \%)$ with an average size of $1.657 \mu \mathrm{m}$, and d) GFP-BSA MPM $(84.3 \%)$ with an average size of $1.25 \mu \mathrm{m}$. For the BSA-GFP solution we got a total $92.7 \%$ of GFP-BSA MPMs with an average size of $1.453 \mu \mathrm{m}$. These results confirm the previous calculation of the Gaussian distribution of particle size

(Figure 3), in which the average size for the major component (BSA-GFP MPM) was $1.406 \mu \mathrm{m} \quad(92.6 \%)$. The same calculations were made for the BSA-(CFP-GBP-YFP) solution (Figure 6), in which the following results were observed: a) pristine BSA microspheres $(4.3 \%)$ with an average size of $2.1 \mu \mathrm{m}$, b) pristine CFP-GBPYFP microspheres $(4.3 \%)$ with an average size of $5.66 \mu \mathrm{m}$, c) reverse (CFP-GBP-YFP)BSA MPMs $(7.1 \%)$ with an average size of $1.5 \mu \mathrm{m}$, and
Figure 3. DLS measurement of MPM size distribution. The average sizes of three examples are: a) $2.34 \mathrm{mi}-$ crons (for BSA microspheres), b) average size for BSA-GFP MPM is $1.406 \mu \mathrm{m}(92.6 \%)$ and $244.0 \mathrm{~nm}(7.4 \%)$ c) average size for BSA-(CFP-GBP-YFP) spheres is $3.525 \mu \mathrm{m}$. 

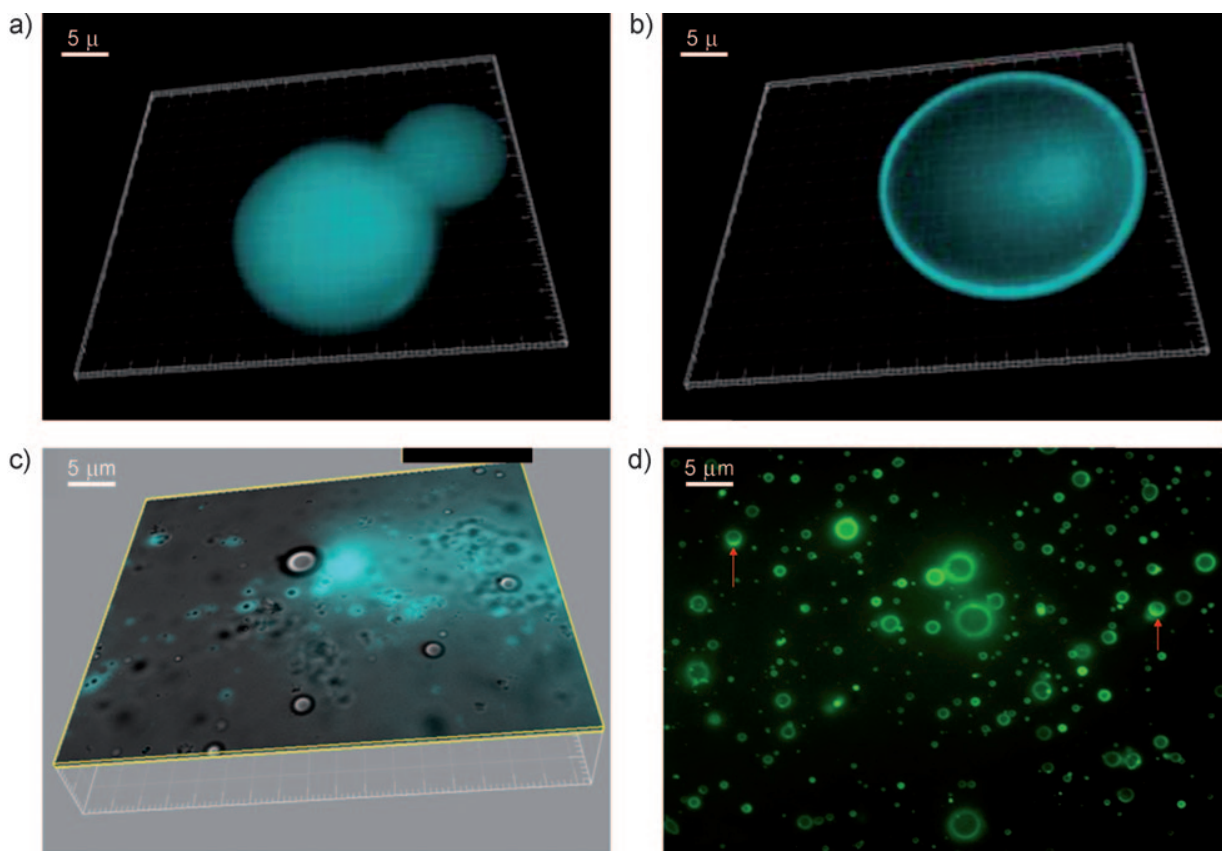

Figure 4. a) The Imaris image for BSA-(CFP-GBP-YFP) MPM. The BSA protein forms the microsphere's walls and the CFP-GBP-YFP protein is encapsulated inside the liquid-filled proteinaceous microsphere bubble. b) The CFP-GBP-YFP protein forms the microsphere's wall. c) The Imaris image with BSA-(CFPGBP-YFP) MPM and one-protein microspheres: BSA-BSA (without a fluorescent signal) and (CFP-GBPYFP)-(CFP-GBP-YFP) (with a strong fluorescent signal in the blue portion of the visible spectrum). d) ApoTome (z-stack) image of BSA-GFP MPM.

a)

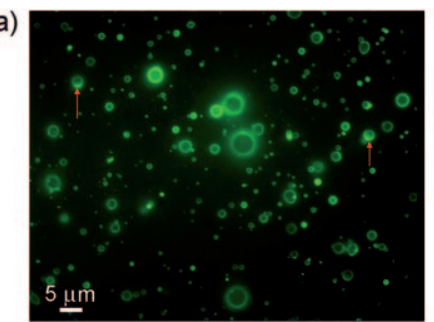

b)

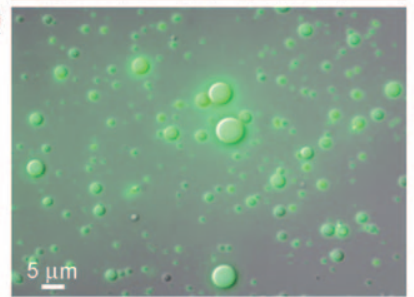

c)
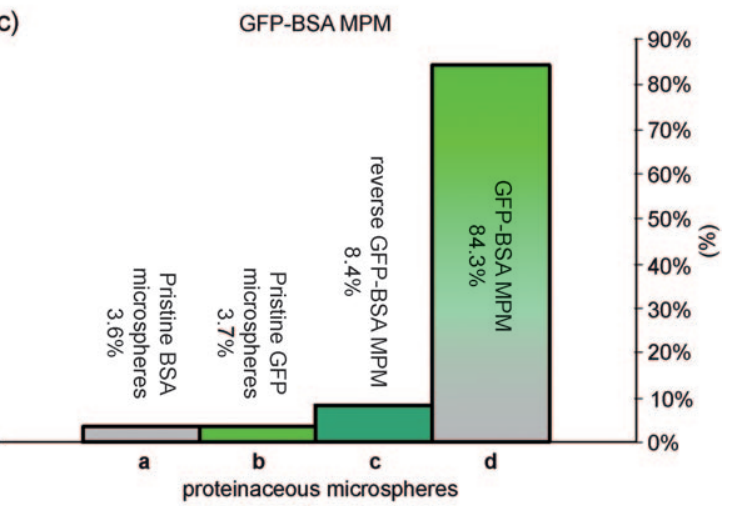

Figure 5. The percentage and size distribution of the various types of the BSA-GFP microspheres. a) ApoTome (z-stack one-color channel) image of BSA-GFP MPM. b) Apo-Tome image of BSA-GFP MPM (a and b are the same images). This figure was used for monitoring the percentage and average size of the pristine BSA microspheres. c) Histogram for abundance of each of the MPM structures. The calculations of the percentage of each of the MPM structures and particle size distribution employed the 'Sicon' image software program: $\mathrm{a}=$ the abundance of pristine BSA microspheres $(3.6 \%)$ with an average size of $2.3 \mu \mathrm{m}, \mathrm{b}=$ pristine GFP microspheres $(3.7 \%)$ with an average size of $435 \mathrm{~nm}, \mathrm{c}=$ reverse GFP-BSA MPMs $(8.4 \%)$ with an average size of $1.657 \mu \mathrm{m}$, and $d=$ GFP-BSA MPM $(84.3 \%)$ with an average size of $1.25 \mu \mathrm{m}$. precursor solution from $2.2: 1$ to 6.6:1 caused an increase in the percentage of pristine BSA microspheres in the resulting solution (from $\sim 3-5$ to $\sim 12 \%$ ). This result is explained by the larger amount of BSA, which will favor the formation of pristine BSA microspheres.

\section{Conclusions}

In this work, we have synthesized mixed proteinaceous microspheres by the sonochemical method. We have proved that there are three possible arrangements that can be obtained from the assembly of two different kinds of proteins in microsphere structures. First, the combined two proteins form the microsphere's walls. Second, the first protein forms the microsphere's walls and the second one is encapsulated inside the liquid-filled proteinaceous microsphere bubble. Third, each kind of protein forms one-protein microspheres. The 'Imaris' images indicated the location of the different proteins in the microsphere structures. We have found that there are differences in the particle's size in the case of oneprotein microspheres against mixed protein microspheres. These differences result from the molecular weight of proteins.

We are currently trying to verify our results by using FPLC measurements. We are also planning to bind antibodies that are specific to CFP-GBPYFP or GFP to the MPM surface to find out the protein percentage in the microsphere walls. d) (CFP-GBP-YFP)-BSA MPM (84.3\%) with an average size of $3.3 \mu \mathrm{m}$.

We have also studied the influence of different ratios of BSA: GFP/(CFP-GBP-YFP) on the composition of the MPM. Increasing the BSA fluorescent protein ratio in the

\section{Experimental Section}

Sonochemical preparation of BSA microspheres: Bovine serum albumin (BSA, molecular weight $66 \mathrm{kDa}$ ), 96-99\% albumin (Sigma) was used 

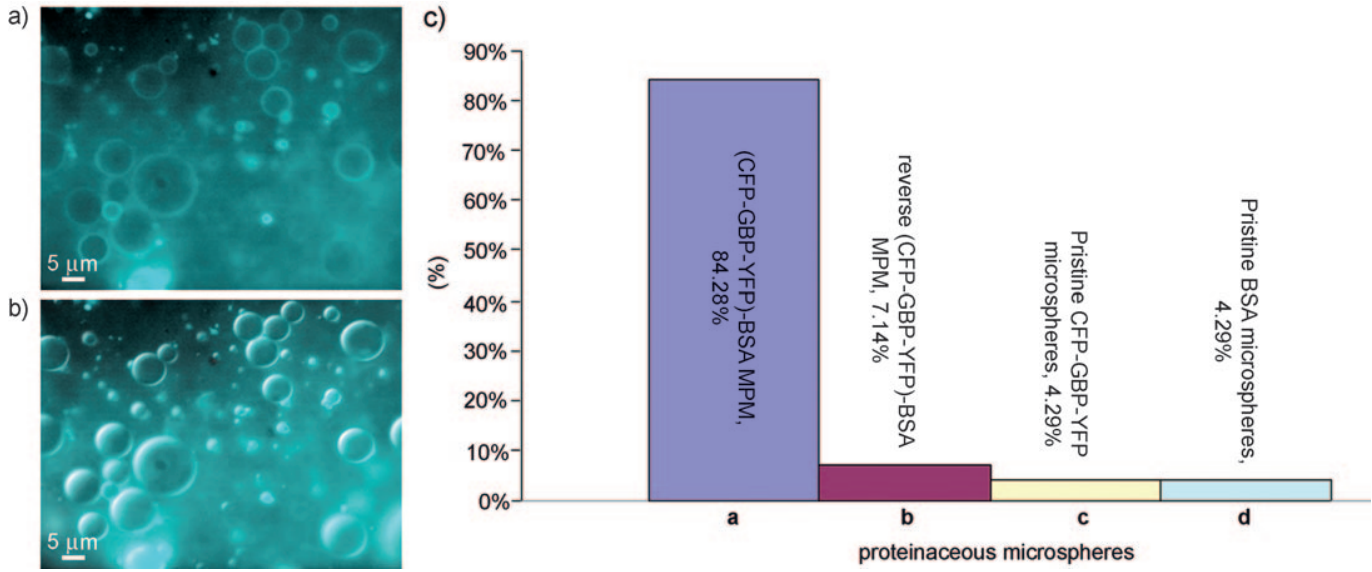

Figure 6. The percentage and size distribution of the various types of the (CFP-GBP-YFP)-BSA microspheres. a) Apo-Tome (z-stack one-color channel) image of BSA-(CFP-GBP-YFP) MPM. b) Apo-Tome image of BSA-(CFP-GBP-YFP) MPM. (Figure 5a and b) are the same images. Figure 5b was used for monitoring the percentage and average size of the pristine BSA microspheres. c) Histogram for abundance of each of the MPM structures. The calculations of the percentage of each of the MPM structures and particle size distribution employed the 'Sicon' image software program. $a=$ the abundance of pristine BSA microspheres $(4.3 \%)$ with an average size of $2.1 \mu \mathrm{m}, \mathrm{b}=$ pristine CFP-GBP-YFP microspheres $(4.3 \%)$ with an average size of $5.66 \mu \mathrm{m}$, $\mathrm{c}=$ reverse (CFP-GBP-YFP)-BSA MPMs $(7.1 \%)$ with an average size of $1.5 \mu \mathrm{m}$, and $\mathrm{d}=($ CFP-GBP-YFP)-BSA MPM $(84.3 \%)$ with an average size of $3.3 \mu \mathrm{m}$.

without any further purification. The preparation of BSA microspheres followed the typical synthesis of proteinaceous microspheres, as described previously. ${ }^{[29]}$ In brief, dodecane $(20 \mathrm{~mL}, 98.0 \%$ Fluka) was layered over a water solution $(30 \mathrm{~mL})$ of $5 \% \mathrm{w} / \mathrm{v}$ protein. The volume ratio between the $5 \%(\mathrm{w} / \mathrm{v})$ BSA aqueous solution and dodecane was kept constant at $3: 2$, respectively.

General conditions for the preparation of MPM: Herein we outline the common conditions used in the preparation of MPMs. MPMs have been synthesized with a high-intensity ultrasonic probe (Sonic and Materials, VC- $600,20 \mathrm{kHz}, 0.5 \mathrm{in}$. Ti horn, at $78 \%$ amplitude). The volume of the acoustic chamber was $25 \mathrm{~mL}$ and the total volume of all the ingredients was $17.7 \mathrm{~mL}$. The bottom of the high-intensity ultrasonic horn was positioned at the aqueous-organic interface, employing an acoustic power of about $150 \mathrm{~W} \mathrm{~cm}^{-2}$ with an initial temperature of $22^{\circ} \mathrm{C}$ in the reaction cell. The sonication lasted for $3 \mathrm{~min}$ at $22^{\circ} \mathrm{C}$ by using an ice-cooling bath to maintain the low temperature. At the end of the reaction, the temperature in the reaction cell reached $28^{\circ} \mathrm{C}$. The rise in temperature from 22 to $28^{\circ} \mathrm{C}$ (measured by a thermocouple) did not change the conformation of the proteins. The temperature in the reaction cell should not rise above the denaturation temperature of the proteins (the recommended temperature is $5{ }^{\circ} \mathrm{C}$ below the denaturation temperature of the proteins). A separation flask was used to separate the product from the mother solution. The separation was accomplished within a few minutes due to the lower density of the microspheres, relative to the density of water. To obtain a more complete separation of the proteinaceous microspheres from the mother solution, the separation flasks were placed in a refrigerator $\left(4^{\circ} \mathrm{C}\right)$ for $24 \mathrm{~h}$. After the separation, the residual aqueous phase and the organic solvent (dodecane) were removed and the product was resuspended in water.

While the GFP was purchased from Alpha-Diagnostic, the CFP-GBPYFP was not purchased from outside sources and was purified according to the following description.

CFP-GBP-YFP purification: A BL21 bacterial E. coli strain $^{[30]}$ transformed with a plasmid expressing the desired protein was induced for protein synthesis. The protein was purified by using a Talon metal affinity resin (Clontech). The CFP-GBP-YFP protein was identified by Western Blot analysis ${ }^{[31]}$ and reveals the presence of the CFP-GBP-YFP protein at $90 \mathrm{kDa} \cdot{ }^{[32]}$

Preparation of BSA-(CFP-GBP-YFP) microspheres: The three starting materials used for the preparation of mixed proteinaceous BSA-(CFPGBP-YFP) microspheres were: 1) a $5 \% \mathrm{w} / \mathrm{v}$ aqueous solution of BSA (bovine serum albumin, 96-99\% Sigma-Aldrich), 2) a CFP-GBP-YFP protein aqueous solution $\left(0.15 \mathrm{mg} \mathrm{mL}^{-1}\right)$, and 3) dodecane $(98.0 \%$ Fluka). The amount of water-soluble CFP-GBP-YFP protein in all the reactions varied from 0.5 to $1.5 \mathrm{~mL}$. The ratio of BSA:(CFP-GBP-YFP) varied from 2.2:1 to 6.6:1. The CFP-GBP-YFP protein was added to the aqueous solution of the BSA. The volume ratio of the $5 \%(\mathrm{w} / \mathrm{v})$ BSA aqueous solution and dodecane was kept constant, $3: 2$, respectively.

Preparation of BSA-GFP microspheres: For the preparation of mixed proteinaceous GFP-BSA microspheres, the following reactants were used: 1 ) a $5 \% \mathrm{w} / \mathrm{v}$ aqueous solution of BSA (bovine serum albumin, 96$99 \%$ Sigma-Aldrich), 2) GFP (recombinant green fluorescent protein; Alpha-Diagnostic), 3) dodecane (98.0\% Fluka). The volume ratio of the $5 \%(\mathrm{w} / \mathrm{v})$ BSA aqueous solution and dodecane was kept constant, $3: 2$, respectively. The GFP protein was added to the aqueous solution of the BSA (ratio BSA: GFP varied from 2.2:1 to 6.6:1).

Characterization methods: See the Supporting Information.

[1] K. S. Suslick, M. W. Grinstaff, J. Am. Chem. Soc. 1990, 112, 78077809.

[2] M. Wong, K. S. Suslick, MRS Symp. Proc. 1995, 372, 89.

[3] N. P. Desai, P. Soon-Shiong, M. W. Grinstaff, Z. Yao, P. A. Sandford, K. S. Suslick, Proc. Soc. Biomaterial. 1994, 20, 112.

[4] B. F. Vandenberg, C. A. Stark, J. A. Rumberger, R. E. Kerber, Am. J. Cardiol. 1988, 62, 333-334.

[5] K. J. Liu, M. W. Grinstaff, J. Jiang, K. S. Suslick, H. M. Swartz, W. Wang, Biophys. J. 1994, 67, 896-901.

[6] J. J. Eckburg, J. C. Chato, K. J. Liu, M. W. Grinstaff, H. M. Swartz, K. S. Suslick, F. P. Auteri, J. Biomech. Eng. 1996, 118, 193-200.

[7] A. G. Webb, M. Wong, K. J. Kolbeck, R. L. Magin, K. S. Suslick, J. Mag. Res. Imaging. 1996, 6, 675-683.

[8] K. S. Suslick, M. W. Grinstaff, Abst. Pap. Am. Chem. Soc. 1994, 207, 257-INOR.

[9] M. W. Grinstaff, K. S. Suslick, Proc. Natl. Acad. Sci. USA 1991, 88, $7708-7710$.

[10] K. S. Suslick, M. W. Grinstaff, K. J. Kolbeck, M. Wong, Ultrason. Sonochem. 1994, 1, S65-S68.

[11] K. S. Suslick, M. W. Grinstaff, Abstr. Pap. Am. Chem. Soc. 1991, 207, 197-POLY.

[12] M. Wong, K. S. Suslick, Abstr. Pap. Am. Chem. Soc. 1995, 210, 587INOR. 
[13] I. Zolle, B. A. Rhodes, J. W. Buchanan, H. N. Wagner, J. Nucl. Med. 1968, 9, 363.

[14] A. J. Bailey, D. N. Rhodes, C. W. Cater, Radiat. Res. 1964, 22, 606.

[15] B. A. Rhodes, I. Zolle, J. W. Buchanan, H. N. Wagner, Radiology 1969, 92, 1453.

[16] S. B. Feinstein, R. M. Lang, C. Dick, A. Neumann, J. Alsadir, K. G. Chua, J. Am. Coll. Cardiol. 1988, 11, 59-65.

[17] S. B. Feinstein, M. W. Keller, C. D. Dick, T. R. Bridenstine, R. W. Wissler, J. Am. Coll. Cardiol. 1987, 9, A111 A111.

[18] M. Wong, K. J. Kolbeck, K. S. Suslick, Abstr. Pap. Am. Chem. Soc. 1994, 207, 79-COLL.

[19] E. Oancea, M. N. Teruel, A. F. G. Quest, T. Meyer, J. Cell Biol. 1998, 140, 485-498.

[20] R. R. Taghizadeh, J. L. Sherley, J. Biomed. Biotechnol. 2008, 453590.

[21] S. Avivi (Levi), A. Gedanken, Biochem. J. 2002, 366, 705-707.

[22] N. C. Shaner, R. E. Campbell, P. A. Steinbach, B. N. G. Giepmans, A. E. Palmer, R. Y. Tsien, Nat. Biotechnol. 2004, 22, 1567-1572.

[23] A. Till, P. Rosenstiel, K. Braeutiqam, C. Sina, G. Jacobs, H. H. Oberg, D. Seegert, T. Chakraborty, S. Schreiber, J. Cell Sci. 2008, 121, 487-495.
[24] J. P. W. Pluim, J. B. A. Maintz, M. A. Viergever, IEEE Trans. Med. Imaging. 2004, 23, 1508-1516.

[25] M. Fend, R. Abt, M. Diefenbacher, S. Bovet, M. Krafft, From Animals to Animats 2004, 8, 114-121.

[26] S. Avivi (Levi), Y. Nitzan, R. Dror, A. Gedanken, J. Am. Chem. Soc. 2003, 125, 15712-15713.

[27] O. Grinberg, M. Hayun, B. Sredni, A. Gedanken, Ultrason. Sonochem. 2007, 14, 661-666.

[28] A. Gedanken, Chem. Eur. J. 2008, 14, 3840-3853.

[29] W. L. Webber, F. Lago, C. Thanos, E. Mathiowitz, J. Biomed. Mater.Res. 1998, 41, 18-29.

[30] T. Yasukawa, C. Kaneiishii, T. Maekawa, J. Fujimoto, T. Yamamoto, S. Ishii, J. Biol. Chem. 1995, 270, 25328-25331.

[31] W. N. Burnette, Anal. Biochem. 1981, 112, 195-203.

[32] F. Luciano, M. Krajewska, P. Ortiz-Rubio, S. Krajewski, D. Y. Zhai, B. Faustin, J. M. Bruey, B. B. Maitre, A. Lichtenstein, S. K. Kolluri, A. C. Satterthwait, X. K. Zhang, J. C. Reed, Blood 2007, 109, 38493855 .

Received: July 28, 2009 Revised: October 19, 2009 Published online: January 11, 2010 